\title{
The Construction of Harmonious Gender Relations Based on Gender Differences
}

\author{
Yu Zhang \\ (Ideological and Political Department, Anhui Sanlian University, Hefei 230001, Anhui province, \\ China) \\ 13499713@qq.com
}

\section{Keywords: Gender differences; Gender relations; Family harmonious; Marriage concept}

\begin{abstract}
The relationship between husband and wife is the core of a family, and harmonious gender relations are the primary factors of family happiness. However, the differences in congenital physiological differences and the acquired education result in the formation of a larger difference between men and women. In real life, if we can not face this difference and effectively solve the resulting conflict, it may lead to that couples can not establish a harmonious relationship between the two sides, thus affecting family happiness. Based on that, this article discussed the differences between the two sexes and how to establish a harmonious relationship in order to give people in contradiction some guidance.

With the development of society, the traditional concept of marriage has made great changes today's, the more prominent thins is growth rate of high divorce rate in the recent. 2015 "Social Service Development Statistics Bulletin" shows that 3.841 million pairs divorced according to the law in our country, the crude divorce rate is $2.78 \%$, for consecutive 12 years. The ratio of marriage to divorce is $27.8 \%$, which means that every 4 pairs of people get marriage at the same time 1 people divorced. The age mainly focus on 30 to 35 years. From 2002 to the present, divorce rate become higher all the way. The Chinese nation has a long tradition of family culture, and in recent years people easily choose to dissolve the existing marriage, breaking the tradition of thousands of years, why? This partial reason is due to the improvement of people's living standards, higher demand of the spiritual life, desiring a happy marriage. But conflict because of gender differences between husband and wife is an important factor in the escalation of contradictions, where the couple do not know the reason of the conflict, do not know how to face and solve the differences, choosing the solution wrong, and ultimately lead to the escalation of war, only parted ways. So what led to the existence of gender differences between men and women?
\end{abstract}

\section{The Reasons Resulting in Gender Differences between Men and Women}

Firstly, because of the society development, since the birth of human society, especially since the patrilineal society, the community has made the division of labor between men and women. Men are mainly engaged in hunting, farming and other more heavy manual labor; women are mainly responsible for domestic work and future generations of reproduction and education. This requires men to withstand greater pressure and conflict. And this kind of labor also affects the development of men's personality and the impact of emotional development. Because woman handle housework and the child's care work in the long-term, this way also affects the female physiological and psychological development and change. Over time, in the long process of human evolution, gender differences has been widely recognized in contemporary society. The so-called gender differences have broad and narrow sense, the broad sense of gender differences refers to the different characteristics in biology, psychology, sociology and other aspects for male and female; narrow gender differences refer to the different characteristics in psychology and sociology. The existence of these differences has brought a positive role in promoting the community to show colorful style, the strong male and female flexibility combined to jointly in the development and progress of society to promote the progress of human society together. However, it is precisely because of this difference, but also led to the emergence of some conflicts in the family life and contradictions, if 
the two sides can respect each other and understand these differences, face up to the resulting conflict, and take effective countermeasures, it will promote the harmony and development of family relations, but if the two sides can not be aware of the differences or understand and accept them, the conflict between the two sides continue to escalate, eventually leading to the breakdown of the marriage and the disintegration of the family.

Secondly, the way of social rearing leads to gender differences between men and women. When the child was born, the gender difference between men and women is not very obvious, before three years old, children do not even have too much sense of difference between men and women. While the community had a great impact on the character of these children after the development to adult. That is, after the child is born, society will give them different gender roles. Gender roles as one of social role, are quality characteristics in line with certain social expectations due to people's gender differences, including gender and different attitudes, personality traits and social behavior patterns. For example, when people educate boys, often consciously told them that boys should be brave, bold; when encounter setbacks, they should learn to self-digest and so on. Over time, the boy learned to silently endure in the virtually, the mood will not want to express, and it seems to be taken for granted in the community. While as to girls, parents will educate them to learn gentle, careful; when faced with difficulties, they can show the weak, they can also express their feelings, but also easy to get adults' attention and care and so on. From the above, it indicates that the community also gives men and women some social gender to some extent, once the performance of these differences not in line with the sex performance, they will not be fully accepted. All these also created men and women to meet the social values in the process of development unconsciously, to join the behavior of the habit recognized by the community, so the difference are formed. This shows that the gender roles of men and women are not born, but form under the influence of various environments in the following day and different cultural atmosphere in the society, personality characteristics and physiological characteristics are not necessarily linked. So what are the differences due to gender differences?

\section{The Main Performance Because of Gender Differences}

First of all, from the brain structure, the connection of left and right brain for men are less, the performance is that they are more sleepy perception of emotion, pay attention to rational thinking, the pursuit of reality, despise emotions. When the conflict occurs, they will try to use a rational way to think, pay attention to solve the problem, when they can not receive the desired effect, they do not know how to meet other in the emotional aspect, so lead to discontent emotions of the other side, then they will choose to escape, drill back to their own pre-set cave healing, the reality will be expressed ignoring on the other's dissatisfaction, or fled the scene; while the brain connection for women are more, showing both women are more emotional, pay attention to feelings. They are willing to take the initiative to establish emotional communication with each other, even if this communication is not very smooth; they also hope that the other side could understand their feelings, rather than concerned about whether the problem is resolved and how to solve. It is the existence of the above differences, in the real relationship between husband and wife, the husband often treat emotion as a part of the whole life, of course, may be a very important part or may also be very light part, this varies from person to person; But the vast majority of women in marriage will put feelings as a very important part of their own lives. Once this part is affected, women are often injured more deeply, because she lost the main part of life, and for most men, it is only an integral part of life, less damage, healing faster. 

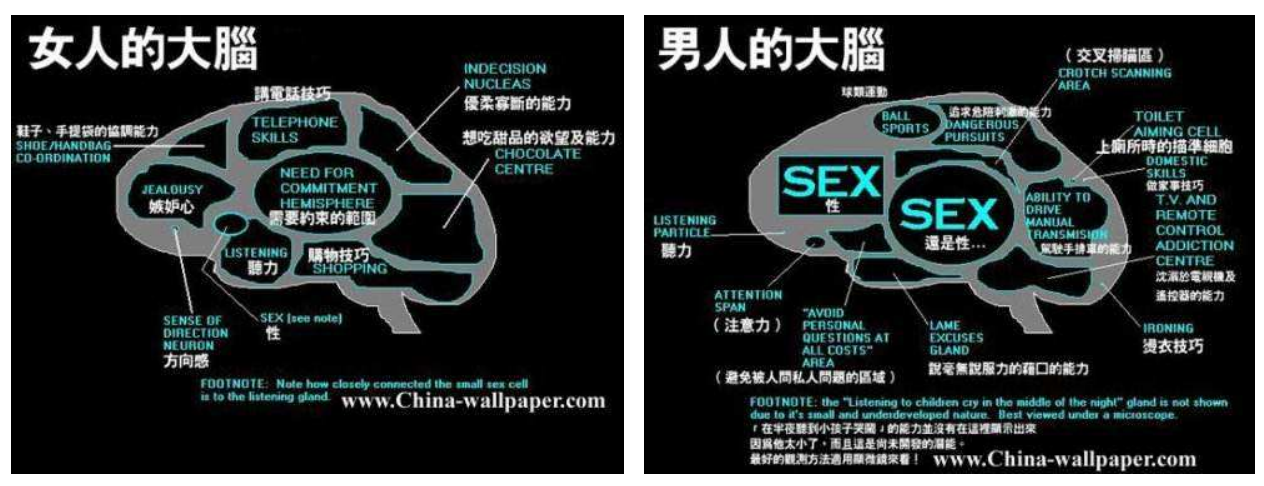

Secondly, from the perspective of the self-worth construction, in the aspect ofsocial culture on gender differences, the concept of gender roles will affect people's perception, attribution, family, health, motivation and behavior, career choice, etc., is an indirect factor causing gender differences. Affected by this, men are often self-centered, building a strong self-esteem by virtue of the success of the cause and the ability. Once this self-esteem is established, they wish to be respected and obeyed; they can be accepted and recognized unconditionally by the other party. They are afraid that others are accused of their incompetence and fear that the business will fail. So they will spend a lot of time in the marriage and effort flutter on the work, and even sacrifice their time to work on the entertainment. Because they know the importance of the business, but also worried about other people's humiliation becaus of the failure of work. In order to show their ability, when others are in trouble, they like unsolicited to help the other side to solve the problem, although they do not like others in the same way to treat him; and they will put their own this ability to the family or friends, to show their power. In fact, this is often easy to lead to their wife's dissatisfaction, because in her view, their relationship is unequal, his wife paid all, but her husband take own "harvest" and the ability to take care of a lot of people. While women are more concerned about the success of the relationship, pay attention to there is a meaningful connection of their relationship with others; love is the first place in the hearts of lover. So they pay more attention to the establishment of equal relations, often more easily concerned about the sense of security. Once there is a sign of trouble, they will be at all to maintain their own safety. It is precisely because they pay more attention to emotion, they want their emotions can be perceived and understood by each other, so when the conflict occurs, they will choose to keep talking, want to be heard and pamper; also hope that the other side as their own could treat the couple's emotional maintenance as the whole of life, and the differences of thought between men and women tend to make women get more hurt in the face of this difference.

Thirdly, in the face of pressure and frustration, men like to get into their own caves, to give yourself enough space not to be disturbed, slowly take decompression, and find the way to solve problem, they seem to be a spring, when they will be pulled back to the limit will rebound back, until the problem is resolved, he will hold a better state to re-enter into the relationship. The same in the face of pressure and frustration, women need to be heard and understood, they are concerned about whether the other side can feel their own pressure and frustration and timely make perception. To the husband and wife life, when the contradictions and quarrels arise, the husband often put themselves "isolated" up, no noise to women, or temporarily escape, waiting until the storm has gone, and then return to the family; and his wife often need to use language to express their dissatisfaction and conflict, when she can not communicate with her husband, she will choose to talk to a third party, this third party may be friends and relatives, it may be friends, but also may be very unreliable "acquaintance", Which fully illustrates the differences on different ways of decompression between men and women in the face of frustration and pressure.

Of course, the difference from the different gender between men and women is far more than these, this article will not repeat them one by one. 


\section{Solutions and Recommendations on Conflict Due to the Gender Differences}

First of all, as a male, in gender relations, they need to learn how to communicate with women, especially in language communication, because the male language is mainly to exchange information, while women is to exchange emotions. Men learn to use language to communicate with women effectively, learn to share their feelings, listen to each other, and found the places worthy of recognition and praise in life, give affirmation and strengthen in a timely manner, so as to give women enough security and self-confidence. In the relationship between husband and wife, as a husband, they need to understand other side, making the other feel love, from the section to start, observe each other's love language and needs, such as often hug each other, prepare some elaborate gifts for each other, which can make woman feel love, and after she feels the love, woman will be completely dedicated themselves in this relationship.

Secondly, as a woman, you need to learn to praise man. As women, especially women in China, they are better at accusations and criticisms, if you want to make the relationship between the two sexes harmonious, women need to break through themselves, learn the way to get along with man, correctly express their needs, affect each other in the words and deeds rather than deliberately change the other side, so that the other occurred in the silent change in the feelings of love. While they need to accept their differences unconditionally, when you need for help, to give a clear instruction, rather than let the other side to guess. In the expression of their own bad mood, they need to be straightforward expression, so as to win the respect and understanding, to eliminate misunderstanding.

And as a modern woman, but also need to learn self-love, developing better myself, concerned about their own needs, and permit to meet your own needs if conditions can be meet. Only in this way, when not concerned or dedication too much, she will not feel unbalanced. In addition, as a woman, she also need to understand the needs of men. Sexual relations in the harmonious relationship is essential, especially when the conflict occurs, must not be sexual threat, because this behavior is likely to lead to the occurrence of extramarital affairs.

Finally, when the conflict occurs, both men and women should learn to focus on the events that cause the conflict itself, effectively control the conflict in the event, rather than an infinite upgrade, leading to deterioration of the relationship. Because most of the time the conflict is often not because of the incident itself, but because of the other's attitude and tone on the incident; to learn to temporarily "truce", such as the choice of isolation law, so that both sides can calm down and then communicate. The two sides also need to always reflect on their own behavior in which the relationship is more conducive to the construction of harmonious relations between the two sides, which is unfavorable, how to overcome them in the future and so on; learn to sympathize with each other, feel each other and learn to give a score for their relation together, and try to raise the score and so on.

\section{Conclusion}

In short, where the differences exist, there the conflict is inevitable, but as long as we can understand differences, respect differences, accept differences, and try to stand at each other's perspective and position to think and resolve differences, harmonious gender relations can be built completely.

\section{Acknowledgements}

This article is the project paper of the Ministry of Education of Humanities and Social Sciences Research Youth Fund, project title: micro-era mainstream ideology micro-transmission mechanism and strategy research, project No.: 16YJC710009

\section{References}

[1] Lu Jie, Wu Ning. Educational Sociology. [M] Beijing: People's Education Press, 2001: 522 
[2] Jin Song, Huang Yongjun. Study on the sociological characteristics of male gender role differences in Teaching [J]. Education and Teaching Research.2009 (9)

[3] (Australia) Malcolm Waters. Modern sociological theory [M]. Beijing: Huaxia Publishing House .2000

[4] Li Xianlin. Some studies on the stereotype of gender roles [J]. Psychological Dynamics .1995, (4): $17-20$

[5] Yan Yu. "Contemporary Chinese marriage ethics evolution and rational orientation research". Jilin Literature and History Publishing House. 2009

[6] "Selected Works of Marx and Engels" (Volume IV); People's Publishing House 2009, 83

[7] Lawrence Stone. English family, sex and marriage [M]. Diao Xiaohua translated. Beijing: Commercial Press, 2011

[8] Daniel. Bell. Capitalist cultural contradictions [M] Yan Beixue translated. Beijing: People's Publishing House 2010

[9] Yan Liping. Analyzing the Chinese and western culture from the perspective of western and China marriage and family concept [J]. Sichuan. Nanchong Reading and Writing Magazine, 2014, 10

[10] Li Cen, Xiong Liying. Engels Marriage and Family Concept [J]. Journal of Economic Research, Heilongjiang. Harbin, 2012, 1 